\title{
Using Atom Probe Tomography as a Forensic Tool to Determine Burnup from Nuclear Fuels
}

\author{
Mukesh Bachhav ${ }^{1 *}$, Jian Gan $^{1}$, Dennis Keiser ${ }^{1}$, Jeffrey Giglio $^{1}$ and Daniel Wachs ${ }^{1}$ \\ 1. Idaho National Laboratory, Idaho Falls, ID, USA. \\ * Corresponding author: mukesh.bachhav@inl.gov
}

Atom Probe Tomography (APT) is routinely employed to determine 3D microstructure and chemistry changes in structural materials used in reactor to understand irradiation effect. These findings help to correlate changes to mechanical and physical properties of materials which are subjected to extreme reactor conditions. Another equally important aspect of nuclear reactors is to be able to predict the changes in the fuel composition and microstructure as a function of burnup for the safety and the economy of a reactor. The burnup calculation is helpful in determining the performance and behavior of fuel during its operation. Also, there is current need to develop advanced fuels with improved properties and performance which requires the experimental data on changes in microstructure, physical and chemical properties after irradiation for feedback process. More often the irradiated microstructure of the fuel is often quite heterogeneous, a reliable method to estimate the local burnup in nuclear fuels with sub-micron high spatial resolution becomes significant for evaluation of the local irradiated microstructure and its correlation to the general behavior of fuels.

A novel approach is presented to determine the local burnup in irradiated fuels using isotopic quantification information obtained by Atom Probe Tomography (APT). This is demonstrated on an asreceived low-enriched (nominal 19.87\%) U-7Mo fuel for enrichment uniformity and on the irradiated fuels at two different burnup levels namely intermediate burnup ( $\sim 52 \%)$ and high burnup ( 69\%). The mass spectrum obtained from all three U-Mo samples is shown in Figure 1. The isotopes of ${ }^{235} \mathrm{U},{ }^{236} \mathrm{U}$, ${ }^{238} \mathrm{U},{ }^{239} \mathrm{Pu}$ and ${ }^{237} \mathrm{~Np}$ for burnup calculation. The expected values are in good agreement with APT calculation which are based on assumption that U-235 fissions with partial loss to U-238 and Pu-239. This method provides local burnup analysis with unprecedented high spatial resolution based on isotopic ratios from as-received and irradiated fuels. APT analysis is also applied to check uranium enrichment and its uniformity for fresh fuel for quality control of fuel fabrication. APT analysis also allowed to investigate the fission products formation and their distribution for irradiated U-Mo fuels which can be directly associated with the burnup levels of the fuel. 

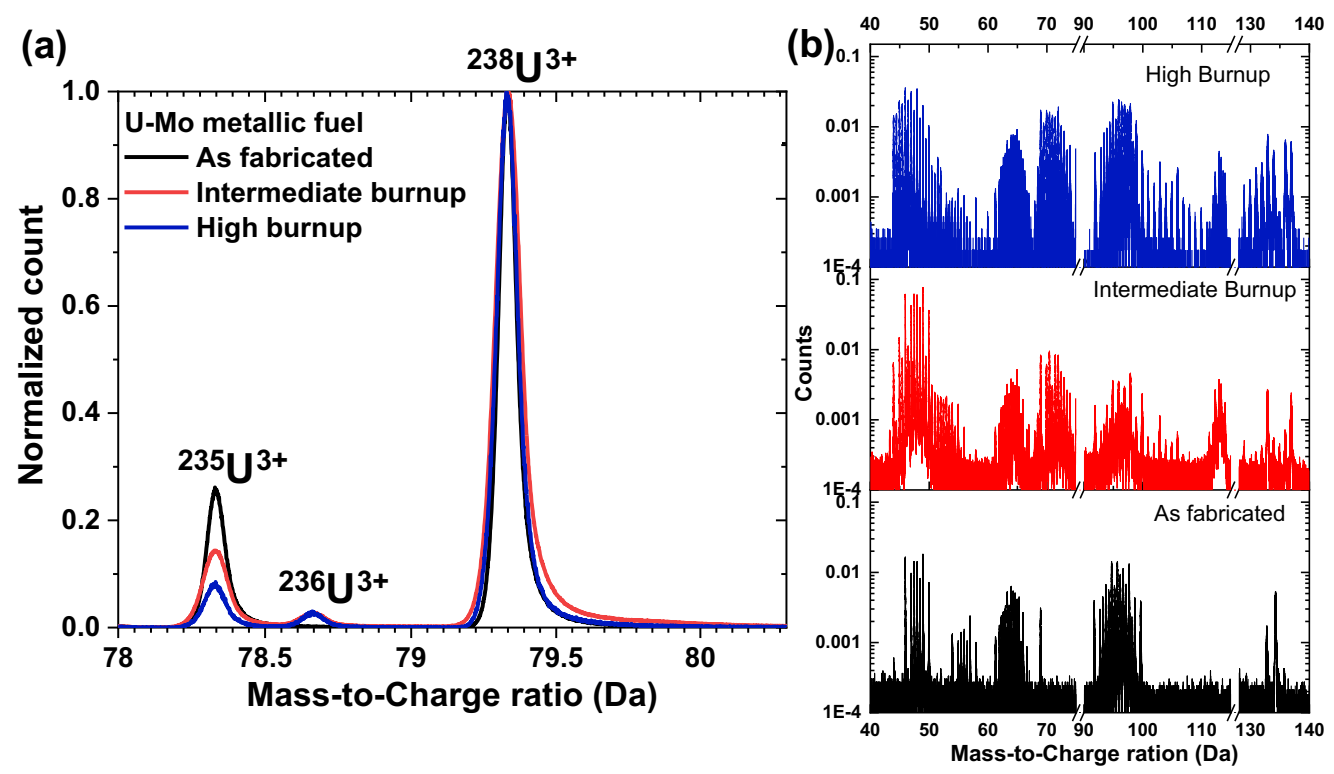

Figure 1. (a) Mass spectrum with different isotopes of $U$ with charge state $U^{3+}$ and (b) fission products (Sr, Xe, Kr, Y, Zr, Nb, Mo, Rc, Ru, Rh, Pd, Ag, Cd, In, Sn, Sb, Te, La, Re, Ce, Pr, Nd, Pm, Sm, Eu, and Gd) obtained from as fabricated (19.87\% enriched), intermediate (52\%) and high (69\%) burnup U-Mo metallic fuel specimen using Atom Probe Tomography. 